TRANSACTIONS OF THE

AMERICAN MATHEMATICAL SOCIETY

Volume 348, Number 8, August 1996

\title{
COMPACT SELF-DUAL HERMITIAN SURFACES
}

\author{
VESTISLAV APOSTOLOV, JOHANN DAVIDOV, AND OLEG MUŠKAROV
}

\begin{abstract}
In this paper, we obtain a classification (up to conformal equivalence) of the compact self-dual Hermitian surfaces. As an application, we prove that every compact Hermitian surface of pointwise constant holomorphic sectional curvature with respect to either the Riemannian or the Hermitian connection is Kähler.
\end{abstract}

\section{INTRODUCTION}

A special feature of oriented Riemannian four-manifolds is the fact that the 2 -vectors can be decomposed into self-dual and anti-self-dual components under the action of the Hodge star operator $*$. As a concequence, the Weyl conformal tensor $\mathcal{W}$ splits into two parts $\mathcal{W}_{+}$and $\mathcal{W}_{-}$defined by $\mathcal{W}_{ \pm}=\frac{1}{2}(\mathcal{W} \pm * \mathcal{W})$. The tensors $\mathcal{W}_{ \pm}$are invariant under conformal changes of the metric, and reversing the orientation of the manifold interchanges their roles. An oriented Riemannian four-manifold $M$ is said to be self-dual (resp. anti-self-dual) if $\mathcal{W}_{-}=0$ (resp. $\left.\mathcal{W}_{+}=0\right)$. It is well-known that the self-duality property plays an important role in the twistor theory since it can be interpreted as the integrability condition for the Atiyah-Hitchin-Singer almost complex structure on the twistor space of $M$ [1].

The classification (up to conformal equivalence) of the compact self-dual manifolds is a very difficult problem which has been solved so far under additional curvature or topological assumptions $[5,6,7,8,9,10,13,15,16,17,19,21,23,24,25,28]$. The main purpose of the present paper is to obtain a classification of the compact self-dual Hermitian surfaces.

If $M$ is a Hermitian surface, its complex structure fixes an orientation on $M$ and this destroys the symmetry between $\mathcal{W}_{+}$and $\mathcal{W}_{-}$. For example, the action of the complex structure on the 2-vectors gives rise to a decomposition of $\mathcal{W}_{+}$whereas $\mathcal{W}_{-}$remains unaffected. In the self-dual case this can be used to obtain, via the Chern-Weil theory and the Miyaoka inequality, useful integral inequalities involving the scalar curvature, the $*$-scalar curvature and the norms of the Lee form and the traceless Ricci tensor of $M$.

The main result in this paper is the following:

Theorem 1. Any compact self-dual Hermitian surface $M$ which is not conformally flat is conformally equivalent either to $\mathbb{C P}^{2}$ with the Fubini-Study metric or to a compact quotient of the unit ball in $\mathbb{C}^{2}$ with the Bergman metric.

Received by the editors December 13, 1994.

1991 Mathematics Subject Classification. Primary 53C55.

Research parially supported by the Bulgarian Ministry of Science and Education, contract MM-423/94. 
This combined with the description of the compact conformally flat Hermitian surfaces due to Ch.Boyer [6, 7] and M.Pontecorvo [24] gives:

Theorem 1'. Any compact self-dual Hermitian surface is conformally equivalent to one of the following:

(i) the complex projective space $\mathbb{C P}^{2}$ with the Fubini-Study metric;

(ii) a compact quotient of the unit ball in $\mathbb{C}^{2}$ with the Bergman metric;

(iii) a complex torus with its flat Kähler metric;

(iv) a hyperelliptic surface with its flat Kähler metric;

(v) $a \mathbb{C P}^{1}$ - bundle over a Riemann surface $\Sigma_{g}$ of genus $g \geq 2$ with the conformally flat Kähler metric which locally is the product of the $(+1)$-curvature metric on $\mathbb{C P}^{1}$ and (-1)-curvature metric on $\Sigma_{g}$;

(vi) a Hopf surface with its conformally flat locally conformally Kähler metric of constant positive scalar curvature.

The proof of Theorem 1 goes as follows. According to a result of Ch.Boyer [6] $\mathrm{M}$ is either a surface of general type or biholomorphically equivalent to $\mathbb{C P}^{2}$. By the solution of the Yamabe problem [27], one can assume that the scalar curvature $\tau$ of $M$ is constant. Now it can be shown, by means of the Miyaoka inequality [22] and an integral formula for the norm of $\mathcal{W}_{+}$(cf. Lemma 3.1), that if $\tau \leq 0$, then $M$ is a Kähler surface of constant negative holomorphic sectional curvature. In the case $\tau>0$, the Gauduchon plurigenera theorem [12] implies that all plurigenera of $M$ vanish (Lemma 3.3). Therefore $M$ is biholomorphic to $\mathbb{C P}^{2}$ (according to the result of Ch.Boyer mentioned above) and it follows, by a result of Y.Poon [25], that $\mathrm{M}$ is conformally equivalent to $\mathbb{C P}^{2}$ with the Fubuni-Study metric.

Theorem $1^{\prime}$ implies the well-known classification result of B.-Y. Chen [8] (cf. also J. P. Bourguignon [5] and A.Derdzinski [9]) that any compact self-dual Kähler surface is one of that listed in the cases (i)-(v) of Theorem $1^{\prime}$.

Theorem $1^{\prime}$ is also used to classify the compact Hermitian surfaces of pointwise constant holomorphic sectional curvature with respect to the Riemannian or the Hermitian connection. Simple local calculations show that these surfaces are selfdual and a case by case examination of the surfaces listed in Theorem $1^{\prime}$ gives the following:

Theorem 2. Every compact Hermitian surface of pointwise constant holomorphic sectional curvature with respect to either the Riemannian or the Hermitian connection is Kähler, so is a complex space form.

In the case of constant non-positive holomorphic sectional curvature Theorem 2 has been proved by Sato \& Sekigawa [26] for the Riemannian connection and by Balas \& Gauduchon [3] for the Hermitian connection. Under the condition that the scalar curvature is constant and non-positive the Riemannian version of this theorem has been recently proved by Sekigawa \& Koda [28]. Theorem 2 and a result of T.Koda [18] (cf. also Lemma 4.1) imply that any compact self-dual Einstein Hermitian surface is a complex space form, a result due to Ch.Boyer [7] and Koda $\&$ Sekigawa [19, 28]. Note that any compact complex space form of dimension 2 is one of the surfaces (i)-(iv) of Theorem $1^{\prime}$. 


\section{ACKNOWLEDGMENT}

We would like to thank P.Gauduchon and M.Pontecorvo for their attention to this work.

\section{Preliminaries}

Let $M=(M, J, g)$ be a Hermitian surface (i.e. a Hermitian manifold of real dimension four) with complex structure $J$ and compatible Riemannian metric $g$. Denote by $\Omega$ the Kähler form of $M$ defined by $\Omega(X, Y)=g(X, J Y)$ for $X, Y \in \chi(M)$ $(\chi(M)$ is the Lie algebra of all smooth vector fields on $M)$. We shall always consider $M$ with the orientation determined by the complex structure $J$. Then the volume form of $M$ is $d V=\frac{1}{2} \Omega \wedge \Omega$. It is well-known (cf. [31]) that $d \Omega=\omega \wedge \Omega$ where $\omega=\delta \Omega \circ J$ is the Lee form of $M$. Note that $M$ is Kähler iff $\omega=0 ; M$ is locally conformally Kähler iff $d \omega=0 ; M$ is conformally Kähler iff $\omega=d \sigma$ for a smooth function $\sigma$ on $M$ (in this case $e^{-\sigma} g$ is a Kähler metric). Let $\nabla, R, \rho$ and $\tau$ be the Riemannian connection, the Riemannian curvature tensor, the Ricci tensor and the scalar curvature of $M$ respectively. (For the curvature tensor we adopt the following definition: $R(X, Y, Z, W)=g\left(\left[\nabla_{X}, \nabla_{Y}\right] Z-\nabla_{[X, Y]} Z, W\right)$.)

Recall that the $*$-Ricci tensor $\rho^{*}$ and the $*$-scalar curvature $\tau^{*}$ of $M$ are defined by

$$
\rho^{*}(X, Y)=\sum_{s=1}^{4} R\left(E_{s}, X, J Y, J E_{s}\right)
$$

and

$$
\tau^{*}=\sum_{s=1}^{4} \rho^{*}\left(E_{s}, E_{s}\right)
$$

where $\left\{E_{s}\right\}$ is a local orthonormal frame of the tangent bundle $T M$. Using the first Bianchi identity, we get

$$
\rho^{*}(X, Y)=-\frac{1}{2} \sum_{s=1}^{4} R\left(X, J Y, E_{s}, J E_{s}\right) .
$$

We also have (cf. [31]):

$$
\tau-\tau^{*}=2 \delta \omega+\|\omega\|^{2} .
$$

Note that on a Kähler manifold the Ricci tensor and the $*$-Ricci tensor coinside; in particular $\tau=\tau^{*}$

The Riemannian metric $g$ induces a metric on the bundle $\wedge^{2}$ of 2-vectors on $M$ by $g\left(X_{1} \wedge X_{2}, X_{3} \wedge X_{4}\right)=\operatorname{det}\left(g\left(X_{i}, X_{j}\right)\right)$. The curvature operator $\mathcal{R}$ is the selfadjoint endomorphism of $\wedge^{2}$ defined by $g(\mathcal{R}(X \wedge Y), Z \wedge W)=-R(X, Y, Z, W)$. The Hodge star operator defines an endomorphism $*: \wedge^{2} \rightarrow \wedge^{2}$ with $*^{2}=I d$. Let $\wedge_{-}^{2}$ and $\wedge_{+}^{2}$ be the subbundles of $\wedge^{2}$ corresponding to the $( \pm 1)$ eigenvalues of $*$. Then $\wedge^{2}=\wedge_{-}^{2} \oplus \wedge_{+}^{2}$. Let $\mathcal{B}=\frac{1}{2}(\mathcal{R}-* \mathcal{R} *) ; \quad \mathcal{W}=\frac{1}{2}(\mathcal{R}+* \mathcal{R} *)-\frac{\tau}{12} I d ; \quad \mathcal{W}_{+}=$ $\frac{1}{2}(\mathcal{W}+* \mathcal{W}) ; \quad \mathcal{W}_{-}=\frac{1}{2}(\mathcal{W}-* \mathcal{W})$. Then

$$
\mathcal{R}=\frac{\tau}{12} I d+\mathcal{B}+\mathcal{W}_{+}+\mathcal{W}_{-}
$$


is the irreducible decomposition of $\mathcal{R}$ under the action of $\mathrm{SO}(4)$ found by Singer and Thorpe [29]. Note that $\mathcal{B}$ and $\mathcal{W}$ represent the traceless Ricci tensor and the Weyl conformal tensor respectively. The manifold $M$ is called self-dual (anti-self-dual) if $\mathcal{W}_{-}=0$ (resp. $\left.\mathcal{W}_{+}=0\right) . M$ is conformally flat iff $\mathcal{W}=0$. It is well-known that the condition $W_{-}=0 \quad\left(W_{+}=0\right)$ is conformally invariant.

Using (2.3) one can obtain the irreducible decomposition of $\mathcal{R}$ under the action of the unitary group $\mathrm{U}(2)$ (cf. [30]). Under this action $\mathcal{B}$ decomposes into two parts $\mathcal{B}_{1}$ and $\mathcal{B}_{2}$ determined as follows:

$$
\mathcal{B}_{1}=\frac{1}{2}(\mathcal{B}+J . \mathcal{B} . J) ; \quad \mathcal{B}_{2}=\frac{1}{2}(\mathcal{B}-J . \mathcal{B} . J) .
$$

Here $J$ is the involution of $\wedge^{2}$ defined by $J(X \wedge Y)=J X \wedge J Y$.

Let $\nabla^{c}$ and $R^{c}$ be the Hermitian connection and the Hermitian curvature tensor of $(M, J, g)$. Denote by $A$ the dual vector field of the 1 -form $\omega$. Then $\nabla^{c}$ and $\nabla$ are related by (cf. [31]):

$$
\nabla_{X}^{c} Y=\nabla_{X} Y-\frac{1}{2} \omega(Y) X-\frac{1}{2} \omega(J X) J Y+\frac{1}{2} g(X, Y) A .
$$

Set $L(X, Y)=\left(\nabla_{X} \omega\right)(Y)+\frac{1}{2} \omega(X) \cdot \omega(Y)$. It is easy to check that

$$
\begin{aligned}
& R^{c}(X, Y, Z, W)=R(X, Y, Z, W)+\frac{1}{2}\{L(X, W) g(Y, Z) \\
& +L(Y, Z) g(X, W)-L(Y, W) g(X, Z)-L(X, Z) g(Y, W)\} \\
& +\frac{\|\omega\|^{2}}{4}\{g(X, Z) g(Y, W)-g(Y, Z) g(X, W)\} \\
& +\frac{1}{2} \Omega(Z, W) \cdot d(\omega \circ J)(X, Y) .
\end{aligned}
$$

Using the fact that $R^{c}(X, Y, Z, W)=R^{c}(X, Y, J Z, J W)$ we get $([31])$ :

$$
\begin{aligned}
& 2\{R(X, Y, Z, W)-R(X, Y, J Z, J W)\}=g(Y, W) L(X, Z) \\
& -g(X, W) L(Y, Z)-g(Y, Z) L(X, W)+g(X, Z) L(Y, W) \\
& +\Omega(Y, Z) L(X, J W)-\Omega(X, Z) L(Y, J W)+\Omega(X, W) L(Y, J Z) \\
& -\Omega(Y, W) L(X, J Z)+\frac{\|\omega\|^{2}}{2}\{g(Y, Z) g(X, W)-g(X, Z) g(Y, W) \\
& -\Omega(Y, Z) \Omega(X, W)+\Omega(X, Z) \Omega(Y, W)\} .
\end{aligned}
$$

Let

$$
\begin{gathered}
\rho_{c}(X, Y)=\sum_{s=1}^{4} R^{c}\left(E_{s}, X, Y, E_{s}\right), \\
\rho_{c}^{*}(X, Y)=-\frac{1}{2} \sum_{s=1}^{4} R^{c}\left(X, J Y, E_{s}, J E_{s}\right)
\end{gathered}
$$

and

$$
2 u=\sum_{s=1}^{4} \rho_{c}^{*}\left(E_{s}, E_{s}\right),
$$




$$
2 v=\sum_{s=1}^{4} \rho_{c}\left(E_{s}, E_{s}\right)
$$

Using (2.6), it is easy to see that

$$
\begin{aligned}
& 2 u=\frac{1}{2}\left(\tau+\tau^{*}\right)+\|\omega\|^{2}, \\
& 2 v=\tau^{*}+\frac{1}{2}\|\omega\|^{2} .
\end{aligned}
$$

Denote by $\chi, p_{1}$ and $c_{1}^{2}$ the Euler characteristic, the first Pontrjagin class and the square of the first Chern class of $M$, respectively. According to the Chern-Weil theory

$$
\begin{gathered}
\chi=\frac{1}{8 \pi^{2}} \int_{M}\left(\left\|\mathcal{W}_{+}\right\|^{2}+\left\|\mathcal{W}_{-}\right\|^{2}+\frac{\tau^{2}}{24}-\|\mathcal{B}\|^{2}\right) d V \\
p_{1}=\frac{1}{4 \pi^{2}} \int_{M}\left(\left\|\mathcal{W}_{+}\right\|^{2}-\left\|\mathcal{W}_{-}\right\|^{2}\right) d V .
\end{gathered}
$$

Taking into account that $c_{1}^{2}=2 \chi+p_{1}$ (cf. [33]) we get

$$
c_{1}^{2}=\frac{1}{4 \pi^{2}} \int_{M}\left(2\left\|\mathcal{W}_{+}\right\|^{2}+\frac{\tau^{2}}{24}-\|\mathcal{B}\|^{2}\right) d V
$$

\section{Proof of Theorem 1}

Let $M=(M, J, g)$ be a compact Hermitian surface. We assume that all tensors are continued by complex linearity. For any orthonormal $J$-frame $\left\{E_{1}, J E_{1}, E_{2}\right.$, $\left.J E_{2}\right\}$, we set

$$
Z_{j}=\frac{1}{\sqrt{2}}\left(E_{j}-i J E_{j}\right) ; \quad Z_{\bar{j}}=\frac{1}{\sqrt{2}}\left(E_{j}+i J E_{j}\right), \quad j=1,2,
$$

and $K_{A B C D}=-R\left(Z_{A}, Z_{B}, Z_{C}, Z_{D}\right)$ where $A, B, C, D \in\{1, \overline{1}, 2, \overline{2}\}$.

Let $\langle$,$\rangle be the Hermitian continuation of g$ on $\wedge^{2} \otimes \mathbb{C}$ and

$$
\begin{aligned}
& \alpha=Z_{1} \wedge Z_{2}, \beta=\frac{1}{\sqrt{2}}\left(Z_{1} \wedge Z_{\overline{1}}+Z_{2} \wedge Z_{\overline{2}}\right), \bar{\alpha}=Z_{\overline{1}} \wedge Z_{\overline{2}}, \\
& \gamma=Z_{1} \wedge Z_{\overline{2}}, \delta=\frac{1}{\sqrt{2}}\left(Z_{1} \wedge Z_{\overline{1}}-Z_{2} \wedge Z_{\overline{2}}\right), \bar{\gamma}=Z_{\overline{1}} \wedge Z_{2} .
\end{aligned}
$$

Then $\{\alpha, \beta, \bar{\alpha}\}$ and $\{\gamma, \delta, \bar{\gamma}\}$ are orthonormal frames of $\wedge_{+}^{2} \otimes \mathbb{C}$ and $\wedge_{-}^{2} \otimes \mathbb{C}$ respectively.

For the proof of Theorem 1 we need two lemmas.

Lemma 3.1. Let $M$ be a compact Hermitian surface. Then

$$
\int_{M}\left\|\mathcal{W}_{+}\right\|^{2} d V=\frac{1}{8} \int_{M}\left\{\|d \omega\|^{2}+\frac{\left(\tau-3 \tau^{*}\right)^{2}}{12}\right\} d V .
$$

Proof. Consider $\mathcal{W}_{+}$as an endomorphism of $\wedge_{+}^{2} \otimes \mathbb{C}$. Then the matrix of $\mathcal{W}_{+}$with respect to the frame $\{\alpha, \beta, \bar{\alpha}\}$ has the following form (cf. [6]):

$$
W_{+}=\left\{\begin{array}{ccr}
\frac{W_{+}^{3}}{W_{+}^{2}} & W_{+}^{2} & W_{+}^{1} \\
\overline{W_{+}^{1}} & -\overline{W_{+}^{2}} & -W_{+}^{2} \\
W_{+}^{2} & W_{+}^{3}
\end{array}\right\} .
$$


Here

$$
\begin{aligned}
& W_{+}^{1}=\langle\mathcal{R} \alpha, \bar{\alpha}\rangle=K_{1212}, \\
& W_{+}^{2}=\langle\mathcal{R} \alpha, \beta\rangle=-\frac{1}{\sqrt{2}}\left\{K_{121 \overline{1}}+K_{122 \overline{2}}\right\}, \\
& W_{+}^{3}=\langle\mathcal{R} \alpha, \alpha\rangle-\tau / 12=K_{12 \overline{12}}-\tau / 12 .
\end{aligned}
$$

Since $\nabla_{X} Y \in T^{1,0} M$ for all $X, Y \in T^{1,0} M$, we have $W_{+}^{1}=K_{1212}=0$. A direct computation involving formula (2.7) shows that

$$
\begin{aligned}
W_{+}^{2}= & -\frac{1}{\sqrt{2}}\left\{K_{1 \overline{1} 12}+K_{2 \overline{2} 12}\right\}=\frac{1}{4 \sqrt{2}}\left\{d \omega\left(E_{1}, E_{2}\right)-d \omega\left(J E_{1}, J E_{2}\right)\right\} \\
& -\frac{i}{4 \sqrt{2}}\left\{d \omega\left(J E_{1}, E_{2}\right)+d \omega\left(E_{1}, J E_{2}\right)\right\} .
\end{aligned}
$$

Since $\delta(\omega \circ J)=0$, we have $d \omega\left(E_{1}, J E_{1}\right)=-d \omega\left(E_{2}, J E_{2}\right)$. Then

$$
\left|W_{+}^{2}\right|^{2} d V=\frac{1}{32}\|d \omega\|^{2} d V+\frac{1}{32} d \omega \wedge d \omega
$$

and, by Stock's theorem, we get

$$
\int_{M}\left|W_{+}^{2}\right|^{2} d V=\frac{1}{32} \int_{M}\|d \omega\|^{2} d V
$$

We also have

$$
\begin{aligned}
\tau & =2\left\{K_{1 \overline{11} 1}+K_{2 \overline{22} 2}+2 K_{12 \overline{12}}+2 K_{1 \overline{212}}\right\}, \\
\tau^{*} & =2\left\{K_{1 \overline{11} 1}+K_{2 \overline{22} 2}-2 K_{12 \overline{12}}+2 K_{1 \overline{12} 2}\right\},
\end{aligned}
$$

and therefore

$$
\tau-\tau^{*}=8 K_{12 \overline{12}} .
$$

Now, by (3.2) and (3.6), we obtain

$$
W_{+}^{3}=\frac{1}{24}\left(\tau-3 \tau^{*}\right)
$$

Hence

$$
\begin{aligned}
\int_{M}\left\|\mathcal{W}_{+}\right\|^{2} d V & =\int_{M}\left(2\left|W_{+}^{1}\right|^{2}+6\left|W_{+}^{3}\right|^{2}+4\left|W_{+}^{2}\right|^{2}\right) d V \\
& =\frac{1}{8} \int_{M}\left\{\|d \omega\|^{2}+\frac{\left(\tau-3 \tau^{*}\right)^{2}}{12}\right\} d V
\end{aligned}
$$

and Lemma 3.1 is proved.

Corollary $3.2([18])$. A compact Hermitian surface is anti-self dual iff $\tau=3 \tau^{*}$ and $d \omega=0$.

Let $M=(M, g)$ be a compact four-dimensional Riemannian manifold. According to the solution of the Yamabe conjecture [27], there is a metric $\widetilde{g}$ in the conformal class of $g$ such that the scalar curvature $\widetilde{\tau}$ of $\widetilde{g}$ is equal to the constant $\lambda(M, g)=$ $\inf \left(\int_{M} \tau^{\prime} d V^{\prime}\right) /\left(\int_{M} d V^{\prime}\right)^{2}$ where the infimum is taken over all metrics $g^{\prime}$ conformally equivalent to $g$. We shall say that $M=(M, g)$ is of positive (resp. zero or negative) type if $\lambda(M, g)$ is positive (resp. zero or negative).

Lemma 3.3. Let $M$ be a compact Hermitian surface of positive type. Then all plurigenera of $M$ vanish. 
Proof. Let $f_{0}$ be the eccentricity function of $M$ defined and studied by Gauduchon $[11,12]$. He has proved that $f_{0}=1$ iff $\delta \omega=0$ and that there always exists a metric in the conformal class of the metric $g$ of $M$ whose eccentricity function is equal to 1 [11, Theorem of Null Eccentricity]. The fundamental constant $C(M, g)$ of $M$ is defined by $C(M, g)=\int_{M} f_{0} u d V$. According to a result of Gauduchon $[12$, Plurigenera Theorem] to prove the lemma it is enough to show that $C(M, g)$ is positive. Since the sign of $C(M, g)$ is a conformal invariant of $g$ (cf. [2, Remark (1.7)]) we can assume that $f_{0}=1$.

Let $\widetilde{g}=e^{-\sigma} g$ be a metric of positive constant scalar curvature $\widetilde{\tau}$. Since $e^{-\sigma} \widetilde{\tau}=$ $\tau-3 \triangle \sigma-\frac{3}{2}\|d \sigma\|^{2}$ (cf. [4]), it follows that

$$
\int_{M} \tau d V=\int_{M}\left(e^{-\sigma} \widetilde{\tau}+\frac{3}{2}\|d \sigma\|^{2}\right) d V>0
$$

Hence, by (2.8) and (2.2), we have

$$
C(M, g)=\int_{M} u d V=\frac{1}{2} \int_{M}\left(\tau+\frac{\|\omega\|^{2}}{2}\right) d V>0
$$

Now we are ready to prove Theorem 1.

Let $M$ be a compact self-dual Hermitian surface which is not conformally flat. Boyer [7, Theorem 5] has shown that in this case either $M$ is a surface of general type or $M$ is biholomorphically equivalent to $\mathbb{C P}^{2}$. Therefore the Miyaoka inequality [22] $c_{1}^{2} \leq 3 \chi$ holds on $M$. Then, from (2.9) and (2.11), we get

$$
\begin{aligned}
0 & \leq 3 \chi-c_{1}^{2}=\frac{1}{8 \pi^{2}} \int_{M}\left(-\|\mathcal{B}\|^{2}-\left\|\mathcal{W}_{+}\right\|^{2}+\frac{\tau^{2}}{24}\right) d V \\
& =\frac{1}{64 \pi^{2}} \int_{M}\left(-8\|\mathcal{B}\|^{2}-\|d \omega\|^{2}+\frac{1}{4}\left(\tau-\tau^{*}\right)\left(\tau+3 \tau^{*}\right)\right) d V
\end{aligned}
$$

By the solution of the Yamabe problem [27], we can assume without loss of generality that the scalar curvature $\tau$ of $M$ is equal to the Yamabe constant $\lambda(M, g)$. Then taking (2.2) into account, the above inequality can be rewriten in the form

$$
0 \leq \frac{1}{64 \pi^{2}} \int_{M}\left(-8\|\mathcal{B}\|^{2}-\|d \omega\|^{2}-\frac{3}{4}\left(\tau-\tau^{*}\right)^{2}+\tau\|\omega\|^{2}\right) d V .
$$

We consider first the case when $M$ is of negative type. Then $\tau<0$ and (3.7) implies $\mathcal{B}=0$ and $\omega=0$. It follows from [17, Theorem 1] (cf. also Lemma 4.1) that $M$ is a compact Kähler surface of constant holomorphic sectional curvature $\frac{1}{6} \tau<0$. Hence $M$ is a compact quotient of the unit ball in $\mathbb{C}^{2}$ with the Bergman metric.

Now suppose that M is of zero type. Then (3.7) gives $d \omega=0$ and $\tau=\tau^{*}=0$. Therefore $\mathcal{W}_{+}=0$ (cf. Corollary 3.2) which contradicts to the assumption that $M$ is not conformally flat.

Finally, let $M$ be of positive type. It follows from Lemma 3.3 that $M$ is not a surface of general type, hence $M$ is biholomorphically equivalent to $\mathbb{C P}^{2}$ according to the result of Boyer mentioned above. Then by a result of Poon [25, Theorem A], we conclude that $M$ is conformally equivalent to $\mathbb{C P}^{2}$ with the Fubinbi-Study metric. Thus the theorem is proved. 


\section{Proof of Theorem 2 for the Riemannian connection}

Lemma 4.1. A Hermitian surface $M$ is of pointwise constant holomorphic Riemannain sectional curvature $c$ iff $\mathcal{B}_{1}=0$ and $\mathcal{W}_{-}=0$. In this case $\tau+3 \tau^{*}=24 c$.

Proof. $M$ is of pointwise constant holomorphic sectional curvature $c$ iff

$$
R(Z, \bar{Z}, Z, \bar{Z})=c .\|Z\|^{4}
$$

for every $Z \in T^{1,0} M$. The latter equality is equivalent to (cf. e.g. [18, Lemma 4.3])

$$
\begin{aligned}
& K_{1 \overline{11} 2}=K_{2 \overline{22} 1}=K_{1 \overline{2} 1 \overline{2}}=0, \\
& K_{1 \overline{1} 1 \overline{1}}=K_{2 \overline{2} 2 \overline{2}}=K_{1 \overline{1} 2 \overline{2}}+K_{1 \overline{2} 2 \overline{1}}=-c .
\end{aligned}
$$

The matrix of $\mathcal{B}_{1}$ with respect to the frame $\{\alpha, \beta, \bar{\alpha}, \gamma, \delta, \bar{\gamma}\}$ defined in Section 3 has the following form

$$
\begin{aligned}
& B_{1}=\left\{\begin{array}{cc}
0 & \mathbf{B}_{1} \\
\overline{\mathbf{B}_{1}^{t}} & 0
\end{array}\right\}, \\
& \mathbf{B}_{1}=\left\{\begin{array}{ccc}
0 & 0 & 0 \\
b_{1} & b_{2} & -\overline{b_{1}} \\
0 & 0 & 0
\end{array}\right\}
\end{aligned}
$$

where

$$
b_{1}=\frac{1}{\sqrt{2}}\left(K_{1 \overline{112}}+K_{2 \overline{212} 2}\right) ; \quad b_{2}=\frac{1}{2}\left(K_{1 \overline{111}}+K_{2 \overline{222}}\right) .
$$

Consider $\mathcal{W}_{-}$as an endomorphism of $\wedge_{-}^{2} \otimes \mathbb{C}$. Then the matrix of $\mathcal{W}_{-}$with respect to the frame $\{\gamma, \delta, \bar{\gamma}\}$ has the following form:

$$
W_{-}=\left\{\begin{array}{ccc}
\frac{W_{-}^{3}}{W_{-}^{2}} & -2 W_{-}^{2} & W_{-}^{1} \\
\overline{W_{-}^{1}} & -\overline{W_{-}^{2}} & W_{-}^{3}
\end{array}\right\} .
$$

Here

$$
\begin{aligned}
& W_{-}^{1}=K_{1 \overline{2} 1 \overline{2}}, \\
& W_{-}^{2}=\frac{1}{\sqrt{2}}\left(-K_{1 \overline{2} 1 \overline{1}}+K_{1 \overline{2} 2 \overline{2}),},\right. \\
& W_{-}^{3}=K_{1 \overline{21} 2}-\tau / 12 .
\end{aligned}
$$

Hence $\mathcal{B}_{1}=0$ and $\mathcal{W}_{-}=0$ iff

$$
\begin{aligned}
& K_{1 \overline{11} 2}=K_{2 \overline{22} 1}=K_{1 \overline{2} 1 \overline{2}}=0, \\
& K_{1 \overline{1} 1 \overline{1}}=K_{2 \overline{2} 2 \overline{2}} ; K_{1 \overline{212} 2}=\tau / 12 .
\end{aligned}
$$

Using (3.4) and the first Bianchi identity we see that (4.4) is equivalent to (4.2). Finally, by (3.6) and (4.3), we get $\tau+3 \tau^{*}=24 c$.

Lemma 4.2. A compact Hermitian surface $M$ is $*$-Einstein iff $\mathcal{B}_{1}=0$ and $d \omega=0$.

Proof. By definition $M$ is $*$-Einstein iff $\rho^{*}(X, Y)=\frac{\tau^{*}}{4} g(X, Y)$. This is equivalent (identifying 2 -vectors with 2-forms) to $\mathcal{R}(\Omega)=\frac{\tau^{*}}{4} \Omega$. Using the frame $\{\alpha, \beta, \bar{\alpha}, \gamma$, $\delta, \bar{\gamma}\}$ we see easily that $M$ is $*$-Einstein iff $\mathcal{B}_{1}=0$ and $W_{+}^{2}=0$. By (3.3) the latter condition is equivalent to $d \omega=0$. 
Lemma 4.3. Let $M=(M, J, g)$ be a compact Kähler surface of constant holomorphic sectional curvature. Then the only Hermitian metrics of pointwise constant holomorphic Riemannian sectional curvature in the conformal class of $g$ are the constant multiples of $g$.

Proof. By Lemmas 4.1 and 4.2, any metric of pointwise constant holomorphic sectional curvature conformal to $g$ is $*$-Einstein. Since every Kähler surface of constant holomorphic sectional curvature is Einstein, the lemma follows from [14, Corollary $3.4]$.

Let $M=(M, J, g)$ be a compact Hermitian surface of pointwise constant holomorphic Riemannian sectional curvature. According to Theorem $1^{\prime}$ we have to consider the following three cases:

1. $M$ is conformally equivalent to a Kähler surface of constant holomorphic sectional curvature. Then by Lemma $4.3 M$ is Kähler.

2. $M$ is a holomorphic $\mathbb{C P}^{1}$ bundle over a complex curve $\Sigma$ of genus $\geq 2$ and $g$ is conformal to the Kähler metric $h$, obtained locally as the product of the metric of constant curvature -1 on the base $\Sigma$ and the metric of constant curvature +1 on the fiber. Denote by $\pi$ the projection $\pi: M \rightarrow \Sigma$. Take local coordinates $\left(z_{1}, z_{2}\right)$ on $M$ such that the metric $h$ has the following form:

$$
h=\frac{4 d z_{1} \otimes d \overline{z_{1}}}{\left(1-\left|z_{1}\right|^{2}\right)^{2}}+\frac{4 d z_{2} \otimes d \overline{z_{2}}}{\left(1+\left|z_{2}\right|^{2}\right)^{2}} .
$$

Then the Kähler form $\Omega$, the Ricci form $\psi$ and the Laplacian $\Delta$ of $M$ are given by

$$
\begin{gathered}
\Omega=-2 i\left(\frac{d z_{1} \wedge d \overline{z_{1}}}{\left(1-\left|z_{1}\right|^{2}\right)^{2}}+\frac{d z_{2} \wedge d \overline{z_{2}}}{\left(1+\left|z_{2}\right|^{2}\right)^{2}}\right), \\
\psi=2 i\left(\frac{d z_{1} \wedge d \overline{z_{1}}}{\left(1-\left|z_{1}\right|^{2}\right)^{2}}-\frac{d z_{2} \wedge d \overline{z_{2}}}{\left(1+\left|z_{2}\right|^{2}\right)^{2}}\right), \\
\Delta=\Delta_{1}+\Delta_{2}
\end{gathered}
$$

where

$$
\Delta_{1}=-\left(1-\left|z_{1}\right|^{2}\right)^{2} \frac{\partial^{2}}{\partial z_{1} \partial \overline{z_{1}}} ; \Delta_{2}=-\left(1+\left|z_{2}\right|^{2}\right)^{2} \frac{\partial^{2}}{\partial z_{2} \partial \overline{z_{2}}}
$$

Let $g$ and $h$ be related by $g=\frac{1}{F^{2}} h$ for some smooth positive function $F$ on $M$. According to Lemmas 4.1 and $4.2, g$ is $*$-Einstein. Now taking into account [14, Theorem 3.3] and the fact that the scalar curvature of $(M, h)$ is equal to zero we obtain

$$
-\triangle F . \Omega=2 F \cdot \psi-4 i \partial \bar{\partial} F .
$$

By (4.5) and (4.6) we have

$$
\Delta_{2} F-\Delta_{1} F=2 F
$$

Differentiating (4.6) we obtain

$$
(-d \triangle F) \wedge \Omega=2 d F \wedge \psi
$$


which in the local coordinates $\left(z_{1}, z_{2}\right)$ can be rewritten as

$$
\begin{aligned}
& \frac{\partial}{\partial z_{1}}(\triangle F+2 F)=\frac{\partial}{\partial \overline{z_{1}}}(\triangle F+2 F)=0, \\
& \frac{\partial}{\partial z_{2}}(\triangle F-2 F)=\frac{\partial}{\partial \overline{z_{2}}}(\triangle F-2 F)=0 .
\end{aligned}
$$

Set

$$
f_{1}=\Delta F-2 F ; \quad f_{2}=\Delta F+2 F .
$$

It follows from (4.8) and (4.9) that

$$
4 F=f_{2}-f_{1} ; \quad \Delta_{1} f_{1}+2 f_{1}=\Delta_{2} f_{2}-2 f_{2}=c
$$

where $c$ is a constant. Then, by (4.7), we obtain that $c=0$, hence

$$
4 F=f_{2}-f_{1} ; \quad \Delta_{1} f_{1}+2 f_{1}=\Delta_{2} f_{2}-2 f_{2}=0 .
$$

Let $p_{0}$ be a point of minimum of $f_{1}$. Take local coordinates $\left(z_{1}, z_{2}\right)$ around $p_{0}$ as above and let in these coordinates $p_{0}=\left(z_{1}^{0}, z_{2}^{0}\right)$. Since, by (4.8), the function $f_{1}$ does not depend on $z_{2},\left(\Delta_{1} f_{1}\right)\left(z_{1}^{0}\right)=\left(\Delta f_{1}\right)\left(z_{1}^{0}, z_{2}^{0}\right) \leq 0$ and, by (4.10), we get $f_{1}\left(z_{1}^{0}\right) \geq 0$. Similar arguments show that there is a point $z_{2}^{*}$ on the fibre of $M$ through $p_{0}$ such that $f_{2}\left(z_{2}^{*}\right) \leq 0$. Then $4 F\left(z_{1}^{0}, z_{2}^{*}\right)=f_{2}\left(z_{2}^{*}\right)-f_{1}\left(z_{1}^{0}\right) \leq 0$, a contradiction.

3. $M$ is conformally equivalent to a Hopf surface with its standard metric $h$. So we can assume that $M=\left(\mathbb{C}^{2}-\{0\}\right) / G$ where $G$ is a group of holomorphic isometries of $\mathbb{C}^{2}-\{0\}$ endowed with the metric $h^{\prime}=\frac{1}{\|z\|^{2}}\left(d z_{1} \otimes d \overline{z_{1}}+d z_{2} \otimes d \overline{z_{2}}\right)$ which acts properly discontinuous and free. Let $\pi: \mathbb{C}^{2}-\{0\} \rightarrow M$ be the natural projection. Then $h^{\prime}=\pi^{*} h$ and $g=\frac{1}{f^{2}} h$ for some smooth positive function $f$ on $M$. By Lemmas 4.1 and 4.2, the metric $g$ is $*$-Einstein, hence $g^{\prime}=\pi^{*} g$ is also a *-Einstein metric. Since $g^{\prime}=\frac{1}{F^{2}} h^{\prime}$ with $F=f \circ \pi$, by [14, Theorem 3.3], we have:

$$
-\triangle(F .\|z\|) . \Omega=-4 i \partial \bar{\partial}(F .\|z\|)
$$

where $\triangle$ and $\Omega$ are respectively the Laplace operator and the Kähler form on $\mathbb{C}^{2}-\{0\}$ with its standard metric $\langle$,$\rangle . Differentiating the latter equality we get$ $\triangle(F .\|z\|)=c=$ const which is equivalent to

$$
\triangle F-\frac{2}{\|z\|}\langle d F, d\|z\|\rangle=\frac{c}{\|z\|}+\frac{F}{\|z\|^{2}} .
$$

It is not hard to see ([32, p. 236]) that every $\gamma \in G$ has the form $\gamma=\rho U$ where $\rho$ is a positive constant and $U$ is an unitary transformation of $\mathbb{C}^{2}$. By a result of Kodaira [20, pp. 694-695], there is $\gamma_{0} \in G$ such that $\lim _{n \rightarrow \infty} \gamma_{0}^{n}(z)=0$ for any point of the unit ball in $\mathbb{C}^{2}$, so the latter condition holds for every point of $\mathbb{C}^{2}$. Let $z^{\prime}$ be a point of global minimum of $F$. Since $F$ is $G$-invariant every $z_{n}^{\prime}=\gamma_{0}^{n}\left(z^{\prime}\right)$ is also a point of minimum of $M$. Then $\Delta F\left(z_{n}^{\prime}\right) \leq 0, d F\left(z_{n}^{\prime}\right)=0$ and it follows from (4.11) that $c\left\|z_{n}^{\prime}\right\|+F\left(z^{\prime}\right) \leq 0$. Taking into account that $\lim _{n \rightarrow \infty} z_{n}^{\prime}=0$, we get $F\left(z^{\prime}\right) \leq 0$, a contradiction. 


\section{Proof of Theorem 2 for the Hermitian connection}

Let $M=(M, J, g)$ be a compact Hermitian surface of pointwise constant holomorphic sectional curvature $k$ with respect to the Hermitian connection. This is equivalent to

$$
R^{c}(Z, \bar{Z}, Z, \bar{Z})=k\|Z\|^{2}
$$

for any $Z \in T^{1.0} M$. Let $\left\{Z_{1}, Z_{\overline{1}}, Z_{2}, Z_{\overline{2}}\right\}$ be the local frame of $T M \otimes \mathbb{C}$ defined at the beginning of Section 3 and let $K_{A B C D}^{c}=-R^{c}\left(Z_{A}, Z_{B}, Z_{C}, Z_{D}\right)$ where $A, B, C, D \in$ $\{1, \overline{1}, 2, \overline{2}\}$. It follows from (5.1) (cf. also [2, Lemma 2.3]) that $M$ is of pointwise constant holomorphic sectional curvature equal to $k$ iff

$$
\begin{aligned}
& K_{1 \overline{1} 1 \overline{2}}^{c}=K_{1 \overline{1} 1 \overline{2}}^{c}+K_{1 \overline{2} 1 \overline{1}}^{c}=K_{1 \overline{2} 2 \overline{2}}^{c}+K_{2 \overline{2} 1 \overline{2}}^{c}=0, \\
& K_{1 \overline{1} 1 \overline{1}}^{c}=K_{2 \overline{2} 2 \overline{2}}^{c}=\frac{1}{2}\left(K_{1 \overline{1} 2 \overline{2}}^{c}+K_{2 \overline{2} 1 \overline{1}}^{c}+K_{2 \overline{1} 1 \overline{2}}^{c}+K_{1 \overline{2} 2 \overline{1}}^{c}\right)=-k .
\end{aligned}
$$

Lemma 5.1. Every Hermitian surface $M$ of pointwise constant holomorphic Hermitian sectional curvature is self-dual.

Proof. The identities (2.7) and (3.8) imply

$$
W_{-}^{1}=K_{1 \overline{2} 1 \overline{2}}^{c} ; W_{-}^{2}=\frac{1}{2 \sqrt{2}}\left(K_{1 \overline{2} 2 \overline{2}}^{c}+K_{2 \overline{2} 1 \overline{2}}^{c}-K_{1 \overline{2} 1 \overline{1}}^{c}-K_{1 \overline{1} 1 \overline{2}}^{c}\right) .
$$

Taking into account (3.4) and the first Bianchi identity we have

$$
\begin{aligned}
W_{-}^{3} & =\frac{1}{6}\left(K_{1 \overline{1} 1 \overline{1}}+K_{2 \overline{2} 2 \overline{2}}-2 K_{1 \overline{1} 2 \overline{2}}-2 K_{1 \overline{1} 2 \overline{1}}\right) \\
& =\frac{1}{6}\left(K_{1 \overline{1} 1 \overline{1}}^{c}+K_{2 \overline{2} 2 \overline{2}}^{c}-K_{1 \overline{1} 2 \overline{2}}^{c}-K_{2 \overline{2} 1 \overline{1}}^{c}-K_{1 \overline{2} 2 \overline{1}}^{c}-K_{2 \overline{1} 1 \overline{2}}^{c}\right)
\end{aligned}
$$

and, by (5.2), we conclude that $\mathcal{W}_{-}=0$.

Lemma 5.2. Let $M=(M, J, g)$ be a compact Kähler surface of constant holomorphic sectional curvature. Then the only Hermitian metrics of pointwise constant holomorphic Hermitian sectional curvature in the conformal class of $g$ are the constant multiples of $g$.

Proof. Let the metric $g^{\prime}=e^{-\sigma} g$ be of pointwise constant holomorphic sectional curvature with respect to the Hermitian connection. According to [3, p. 42]

$$
c_{1}^{2}=\frac{1}{8 \pi^{2}} \int_{M} u^{\prime} \cdot v^{\prime} d V^{\prime}
$$

where $u^{\prime}$ and $v^{\prime}$ are the scalar curvatures of $g^{\prime}$ defined in Section 2. On the other hand $(M, J, g)$ is Kähler-Einstein, hence $c_{1}^{2}=\frac{1}{32 \pi^{2}} \int_{M} \tau^{2} d V$. Taking into account that $2 u=2 v=\tau=\tau^{*}$ (cf. (2.8)) we obtain:

$$
\int_{M} u^{\prime} v^{\prime} d V^{\prime}=\int_{M} u^{2} d V
$$

It is well-known (cf. [2]) that $u^{\prime}, v^{\prime}$ and $u$ are related by

$$
e^{-\sigma} u^{\prime}=u-2 L(\sigma) ; \quad e^{-\sigma} v^{\prime}=u-L(\sigma)
$$

where $L$ is the complex Laplacian given in local coordinates by

$$
L=-\sum_{\alpha, \bar{\beta}} g^{\alpha \bar{\beta}} \frac{\partial^{2}}{\partial z_{\alpha} \partial z_{\bar{\beta}}} .
$$


Then

$$
\int_{M}(u-2 \cdot L(\sigma)) \cdot(u-L(\sigma)) d V=\int_{M} u^{2} d V .
$$

Using the fact that $u=$ const and $\int_{M} L(\sigma) d V=0$, we conclude that $L(\sigma)=0$ and it follows from the maximum principle that $\sigma=$ const.

Now we are ready to prove Theorem 2 for the Hermitian connection. Let $M=(M, J, g)$ be a compact Hermitian surface of pointwise constant holomorphic Hermitian sectional curvature. Then from Lemma 5.1 we infer that $M$ is self-dual and according to Theorem 1 we have to consider the following two cases:

1. $M$ is conformally equivalent to $\mathbb{C P}^{2}$ with the Fubini-Study metric or to a compact quotient of the unit ball in $\mathbb{C}^{2}$ with the Bergman metric. Then, by Lemma 5.2, $M$ is Kähler.

2. $M$ is conformally flat. According to $[6$, Theorem 1,3$]$ we have $c_{1}^{2} \leq 0$. On the other hand, by Corollary $3.2, \tau=3 \tau^{*}$ which is equivalent to $u=2 v$. Then, by (5.3), we obtain $c_{1}^{2}=\frac{1}{4 \pi^{2}} \int_{M} v^{2} d V \geq 0$. So $c_{1}^{2}=0$ and $u=v=0$. Then $\int_{M}(u-v) d V=\frac{1}{2} \int_{M}\|\omega\|^{2} d V=0$ (cf. (2.2) and (2.8)). Hence $\omega=0$, i.e. $M$ is Kähler.

\section{REFERENCES}

1. M.F.Atiyah, N.J.Hitchin, I.M.Singer, Self-duality in four-dimensional Riemannian geometry, Proc.Roy.Soc. London Ser.A 362 (1978), 425-461. MR 80d:53023

2. A.Balas, Compact Hermitian Manifolds of Constant Holomorphic Sectional Curvature, Math.Z. 189 (1985), 193-210. MR 86f:53072

3. A.Balash, P.Gauduchon, Any Hermitian Metric of Constant Non-Positive (Hermitian) Holomorphic Sectional Curvature is Kähler, Math.Z. 190 (1985), 39-43. MR 86h:53066

4. A.Besse, Einstein Manifolds, Ergebnisse der Mathematik und ihrer Grenzgebiete 3.Folge, Band 10, Springer, Berlin Heildelberg New-York, 1987. MR 88f:53087

5. J.P.Bourguignon, Les variétés de dimension 4 à signature non nule dont la courbure est harmonique sont d'Einstein, Invent. Math. 63 (1981), 263-286. MR 82g:53051

6. Ch.Boyer, Conformal duality and compact complex surfaces, Math. Ann. 274 (1986), 517-526. MR 87i:53068

7. Ch.Boyer, Self-dual and anti-self-dual Hermitian metrics on compact complex surfaces, Mathematics and general relativity, Proceedings, Santa Cruz 1986 (J.A.Isenberg, ed.), Contemp.Math., vol. 71, AMS, Providence, 1988, pp. 105-114. MR 89h:53127

8. B.-Y.Chen, Some topological obstructions to Bochner-Kähler metrics and their applications, J.Diff.Geom. 13 (1978), 574-588. MR 81f:32037

9. A.Derdzinski, Self-dual Kähler manifolds and Einstein manifolds of dimension four, Compositio Math. 49 (1983), 405-433. MR 84h:53060

10. Th.Friedrich, H.Kurke, Compact four-dimensional self-dual Einstein manifolds with positive scalar curvature, Math.Nachr. 106 (1982), 271-299. MR 84b:53043

11. P.Gauduchon, Le théorème de l'excentricité nulle, C.R.Acad.Sci.Paris, Ser.A 285 (1977), 387-390. MR 57:10664

12. P.Gauduchon, Fibrés Hermitiens à endomorphisme de Ricci non négatif, Bull.Soc.Math. France 105 (1977), 113-140. MR 58:6375

13. P.Gauduchon, Self-dual manifolds with non-negative Ricci operator, Prospects in complex geometry, Proceedings, Katata and Kyoto 1989 (J.Noguchi, T.Ohsawa, eds.), Lecture Notes in Math., vol. 1468, Springer, Berlin Heidelberg New York, 1991, pp. 55-61. MR 94g:53032

14. G.Grantcharov, O.Muškarov, Hermitian *-Einstein surfaces, Proc.Amer.Math.Soc. 120 (1994), 233-239. MR 94b:53081

15. N.J.Hitchin, Compact four-dimensional Einstein manifolds, J.Diff.Geom. 9 (1974), 435-441. MR 50:3149 
16. N.J.Hitchin, Kählerian twistor spaces, Proc.London Math.Soc. 43 (1981), 133-150. MR 84b:32014

17. M.Itoh, Self-duality of Kähler surfaces, Compositio Math. 51 (1984), 265-273. MR 85m:53079

18. T.Koda, Self-dual and anti-self-dual Hermitian surfaces, Kodai Math. J. 10 (1987), 335-342. MR 89a:53053

19. T.Koda, K.Sekigawa, Self-dual Einstein Hermitian surfaces, Progress in Diff.Geom. (K.Shiohama, ed.), Advanced Studies in Pure Mathematics, vol.22, Kinokuniya, Tokyo, 1993, pp. 123-131. MR 95b:53056

20. K.Kodaira, On the structure of compact complex analytic surfaces, II, Amer.J.Math. 88 (1968), 682-721. MR 34:5112

21. J.Lafontaine, Remarks sur les variété conformément plates, Math.Ann. 259 (1982), 313-319. MR 84a:53053

22. Y.Miyaoka, On Chern numbers of surfaces of general type, Invent.Math. 42 (1977), 225-237. MR 57:337

23. M.H.Noronha, Self-duality and 4-manifolds with nonnegative curvature on totally isotropic 2-planes, Michigan Math.J. 41 (1994), 3-12. MR 95e:53069

24. M.Pontecorvo, Uniformization of conformally flat Hermitian surfaces, Diff.Geom. and its Appl. 2 (1992), 295-305. MR 94k:32052

25. Y.Poon, Compact self-dual manifolds with positive scalar curvature, J.Diff.Geom. 24 (1986), 97-132. MR 88b:32022

26. T.Sato, K.Sekigawa, Hermitian surfaces of constant holomorphic sectional curvature, Math.Z. 205 (1990), 659-668. MR 91m:53052

27. R.Schoen, Conformal deformations of Riemannian metrics to constant scalar curvature, J.Diff.Geom. 20 (1984), 479-495. MR 86i:58137

28. K.Sekigawa, T.Koda, Compact Hermitian surfaces of pointwise constant holomorphic sectional curvature, to appear in Glasgow Math.J.

29. I.M.Singer, J.A.Thorpe, The curvature of 4-dimensional Einstein spaces, Global analysis, Papers in honor of K.Kodaira (D.C.Spencer, S.Iyanaka, eds.), Princeton University Press, Princeton, 1969, pp. 355-365. MR 41:959

30. F.Tricerri, L.Vanhecke, Curvature tensors on almost-Hermitian manifolds, Trans. Amer. Math. Soc. 267 (1981), 365-398. MR 82j:53071

31. I.Vaisman, Some curvature properties of complex surfaces, Ann.Mat.Pura Appl. 32 (1982), 1-18. MR 84i:53064

32. I.Vaisman, Generalized Hopf manifolds, Geom. Dedicata 13 (1982), 231-255. MR 84g:53096

33. W.T.Wu, Sur la structure presque complex d'une variété différentiable réelle de dimension 4, C.R.Acad.Sci.Paris 227 (1948), 1076-1078. MR 10:318b

Institute of Mathematics, Bulgarian Academy of Sciences, Acad. G. Bonchev St. BL.8, 1113 Sofia, Bulgaria

E-mail address: jtd@bgearn.bitnet 